\title{
\begin{tabular}{l|l} 
Mitraries & DSpace@MIT
\end{tabular}
}

\author{
MIT Open Access Articles
}

\section{AROUND 200 NEW X-RAY BINARY IDS FROM 13 YR OF CHANDRA OBSERVATIONS OF THE M31 CENTER}

The MIT Faculty has made this article openly available. Please share how this access benefits you. Your story matters.

Citation: Barnard, R., M. R. Garcia, F. Primini, Z. Li, F. K. Baganoff, and S. S. Murray. "AROUND 200 NEW X-RAY BINARY IDs FROM 13 YR OF CHANDRA OBSERVATIONS OF THE M31 CENTER." The Astrophysical Journal 780, no. 1 (December 13, 2013): 83. (C) 2014 The American Astronomical Society

As Published: http://dx.doi.org/10.1088/0004-637x/780/1/83

Publisher: IOP Publishing

Persistent URL: http://hdl.handle.net/1721.1/95433

Version: Final published version: final published article, as it appeared in a journal, conference proceedings, or other formally published context

Terms of Use: Article is made available in accordance with the publisher's policy and may be subject to US copyright law. Please refer to the publisher's site for terms of use. 


\title{
AROUND 200 NEW X-RAY BINARY IDs FROM 13 YR OF CHANDRA OBSERVATIONS OF THE M31 CENTER
}

\author{
R. Barnard ${ }^{1}$, M. R. Garcia ${ }^{1}$, F. Primini ${ }^{1}$, Z. Li ${ }^{2}$, F. K. BaganofF ${ }^{3}$, And S. S. MurraY ${ }^{4}, 1$ \\ ${ }^{1}$ Harvard-Smithsonian Center for Astrophysics, Cambridge, MA 02138, USA \\ ${ }^{2}$ School of Astronomy and Space Science, Nanjing University, Nanjing 210093, China \\ ${ }^{3}$ Kavli Institute for Astrophysics and Space Research, Massachusetts Institute of Technology, Cambridge, MA 02139, USA \\ ${ }^{4}$ Johns Hopkins University, Baltimore, MD, USA \\ Received 2013 June 17; accepted 2013 November 7; published 2013 December 13
}

\begin{abstract}
We have created $0.3-10 \mathrm{keV}, 13 \mathrm{yr}$, unabsorbed luminosity lightcurves for $528 \mathrm{X}$-ray sources in the central $20^{\prime}$ of M31. We have 174 Chandra observations spaced at $\sim 1$ month intervals due to our transient monitoring program, deeper observations of the M31 nucleus, and some public data from other surveys. We created $0.5-4.5 \mathrm{keV}$ structure functions (SFs) for each source for comparison with the ensemble SF of active galactic nuclei (AGN). We find 220 $\mathrm{X}$-ray sources with luminosities $\gtrsim 10^{35} \mathrm{erg} \mathrm{s}^{-1}$ that have SFs with significantly more variability than the ensemble AGN SF, and which are likely X-ray binaries (XBs). A further $30 \mathrm{X}$-ray sources were identified as XBs using other methods. We therefore have 250 probable XBs in total, including $\sim 200$ new identifications. This result represents great progress over the $\sim 50 \mathrm{XBs}$ and $\sim 40 \mathrm{XB}$ candidates previously identified out of the $\sim 2000 \mathrm{X}$-ray sources within the $D_{25}$ region of M31; it also demonstrates the power of SF analysis for identifying XBs in external galaxies. We also identify a new transient black hole candidate, associated with the M31 globular cluster B128.
\end{abstract}

Key words: galaxies: individual (M31) - methods: data analysis - stars: black holes - X-rays: binaries X-rays: general

Online-only material: color figures, machine-readable tables

\section{INTRODUCTION}

The X-ray populations of external galaxies have the potential to provide excellent diagnostics on the evolutionary states of their host galaxies. However, separating the true galaxy population from active galaxies in the field is notoriously difficult. This is because $\mathrm{X}$-ray binaries (XBs) are expected to dominate the X-ray populations of galaxies, and their emission spectra can be very similar to the spectra of active galactic nuclei (AGN). In fact, it has been rather difficult to classify $\mathrm{X}$-ray sources in external galaxies at all; Stiele et al. (2011) examined $1897 \mathrm{X}$-ray sources within the $D_{25}$ region for M31 but only identified $46 \mathrm{XBs}$ and $43 \mathrm{XB}$ candidates, while $\sim 65 \%$ of the X-ray sources had no classification. However, we have recently invented a method for discriminating between XBs and AGN by formalizing their differences in variability (Barnard et al. 2012b).

It has long been known that AGN may vary by a factor of 2-3 on timescales of months to years, with the amplitude of variation inversely proportional to the luminosity (see, e.g., Marshall et al. 1981; Nandra et al. 1997 and references within). However, exceptional AGN can flare up by an order of magnitude (e.g., Tananbaum et al. 1978).

Recently, Vagnetti et al. (2011) studied the ensemble variability over timescales of hours to years of AGN in the serendipitous source catalogs from XMM-Newton (Watson et al. 2009) and Swift (Puccetti et al. 2011); their sample covered redshifts $\sim 0.2-4.5$ and $0.5-4.5 \mathrm{keV}$ luminosities $\sim 10^{43}-10^{46} \mathrm{erg} \mathrm{s}^{-1}$. They included 412 AGN from the XMM-Newton catalog and 27 AGN from the Swift catalog; all of these AGN were sampled at least twice. They used a structure function (SF) to estimate the mean intensity deviation for data separated by time $\tau$ :

$$
\mathrm{SF}(\tau) \equiv \sqrt{\frac{\pi}{2}\left\langle\left|\log f_{\mathrm{X}}(t+\tau)-\log f_{\mathrm{X}}(t)\right|\right\rangle^{2}-\sigma_{n}^{2}}
$$

where $\sigma_{n}$ is the photon noise and $f_{\mathrm{X}}$ is the $\mathrm{X}$-ray flux. They grouped the SF into logarithmic bins with width 0.5 ; each bin in the range $\log (\tau)=0.0-3.0$ contained more than 100 measurements.

Vagnetti et al. (2011) found good agreement between the $X M M-N e w t o n$ and Swift samples of AGN, after the noise components were subtracted: $\mathrm{SF}(\tau) \propto \tau^{0.10 \pm 0.01}$ for the $X M M$ Newton sample and $\operatorname{SF}(\tau) \propto \tau^{0.07 \pm 0.04}$ for the Swift sample. They also investigated the well-observed anticorrelation between intensity variability $\left(I_{\mathrm{var}}\right)$ and luminosity, expressed in the form $I_{\text {var }} \propto L_{\mathrm{X}}^{-k} ; k \sim 0.3$ in the literature for timescales of days to tens of days. They measured $k$ for AGN grouped logarithmically over $\log L_{X}=43.5-45.5$ for two values of $\tau: 1$ day and 100 days. They find $k=0.42 \pm 0.03$ for $\tau=1$ day, and $k=0.21 \pm 0.07$ for $\tau=100$ days; the latter result is consistent with the work of Markowitz \& Edelson (2004), who found $k \sim 0.13$ for variations over year-long timescales.

Recently, Stiele et al. (2008) examined the variability of Xray sources in the M31 center over nine deep XMM-Newton observations spanning from 2000 June to 2004 July; they obtained fluxes or upper limits for each X-ray source in every observation and searched for differences between maximum and minimum flux $>3 \sigma$. They found 149 sources out of 300 that exhibited $>3 \sigma$ flux variability; of those, 44 exhibited variability by a factor $>5$, including 28 XBs or XB candidates. Stiele et al. (2008) reclassified any "hard" X-ray source that varied by a factor $>10$ as a candidate $\mathrm{XB}$; however, according to the SF obtained by Vagnetti et al. (2011), typical AGN only vary by $\lesssim 70 \%$ over four years, meaning that further XBs could have been identified in hindsight from that variability study.

We have been studying the long-term variability of 528 $\mathrm{X}$-ray sources over 174 Chandra observations of the inner regions of M31, spanning $\sim 13 \mathrm{yr}$. We conducted a pilot SF survey on $37 \mathrm{X}$-ray sources associated with globular clusters that are probable low-mass XBs (LMXBs; Barnard et al. 
2012b). We found that the lower luminosity XBs tended to exhibit considerably more variability than the ensemble AGN SF; the higher luminosity XBs exhibited SFs that indicated comparable or lesser variability than the ensemble AGN SF. Such dependence on variation with luminosity is well known in Galactic systems, where the Z-sources (high luminosity XBs) vary only by a factor of a few, while the atoll sources (low luminosity XBs) vary by $\sim 1-2$ orders of magnitude (Muno et al. 2002).

In this work we will present an overview of the long-term variability of the $528 \mathrm{X}$-ray sources in our chosen region, out to $20^{\prime}(\sim 4.5 \mathrm{kpc})$ from the center of M31. We have created 0.5-4.5 keV SFs for each target for comparison with the ensemble AGN SF created by Vagnetti et al. (2011). With these results, we will place limits on the numbers of XBs and AGN in this region. We will also compare the number of possible AGN with the expected number from the $0.5-10 \mathrm{keV}$ AGN flux distribution created by Georgakakis et al. (2008).

We have previously published detailed studies of 13 X-ray transients with Chandra and Hubble Space Telescope coverage (Barnard et al. 2012a, 2013b), and of those X-ray sources associated with M31 globular clusters (GCs; Barnard et al. 2012 b). We also recently published our 26 new black hole candidates (BHCs) that we identified via SF analysis (Barnard et al. 2013a). Further work on the $\sim 50$ transients in our study will be presented separately (R. Barnard et al., in preparation). In the following sections, we provide details of the observations and data reduction, followed by results and our discussion.

\section{OBSERVATIONS AND DATA REDUCTION}

The central region of M31 has been observed with Chandra on a $\sim$ monthly basis for the last $\sim 13 \mathrm{yr}$ in order to monitor transients; we exclude periods when M31 cannot be viewed due to orbital constraints (approximately March-May each year). We have analyzed 112 ACIS observations and 62 HRC observations, in order to discern the variability of X-ray sources in this region.

Initial source detection was performed on a merged ACIS events file created from observations performed from 1999 October to 2010 March; the detection procedure followed that outlined by Wang (2004). Each observation was registered to a single coordinate system, with the systematic uncertainty in registration included in the uncertainty of the centroid position in the merged image. The total exposure was $305 \mathrm{ks}$ but the combined effective $0.5-8 \mathrm{keV}$ exposure varied considerably over the field of view, from $\sim 2 \times 10^{5} \mathrm{~s}$ in the center to $\sim 1.1 \times 10^{5} \mathrm{~s}$ within $6^{\prime}$ of the center, and down to $\sim 10^{4} \mathrm{ks}$ at larger off-axis angles. The highest sensitivity was found $\sim 2^{\prime}$ from the nucleus $\left(\sim 7 \times 10^{-5}\right.$ count s$^{-1}$, or $\left.\sim 5 \times 10^{34} \mathrm{erg} \mathrm{s}^{-1}\right)$; diffuse emission is highly significant within $2^{\prime}$, while a combination of degrading point-spread functions (PSFs) and lower exposures decreased the sensitivity by a factor $\gtrsim 20$ at high offaxis angles.

A source was considered significant if the best fit line of constant intensity was $\geqslant 3 \sigma$ above zero. Some transient X-ray sources would be rejected by this criterion, so we also accepted sources that demonstrated significant variability.

We compared our initial Chandra source list with the inventory from an XMM-Newton survey of M31 performed by Stiele et al. (2011). We found that the XMM-Newton catalog contained sources that were not in our initial sample. We obtained lightcurves for each of those sources and any that were significantly detected in our observations were added to the source list.

We determined the position of each source from a merged 0.3-7 keV ACIS image, using the IRAF tool IMCENTROID. This merged image is registered to the $B$ band image of M31 Field 5 in the Local Group Survey (LGS) of Massey et al. (2006) using 27 X-ray-bright GCs, as described in Barnard et al. (2012a). For transients that were more recent than the merged Chandra image, we registered the Chandra observation with the highest $\mathrm{X}$-ray flux for that transient to the merged Chandra image. For most sources, the position uncertainties combine the systematic uncertainty in registering the merged Chandra image to the Field $5 B$ band image and the statistical uncertainty in the position of the X-ray centroid; new transients have an additional uncertainty in registering the peak observation to the merged image.

We obtained 0.3-7.0 keV spectra from the circular source and background regions for each source. The background region was the same size as the source region and at a similar off-axis angle. The extraction radius varied between sources, because larger off-axis angles resulted in larger PSFs.

\subsection{Converting from Intensity to Luminosity}

We used XSPEC to convert from $0.3-7.0 \mathrm{keV}$ intensity to 0.3-10 keV luminosity for each observation of every source. Rather than assuming a single conversion factor for a particular emission model, we calculated the conversion factor at the location of each X-ray source in every observation; this was necessary because any given source could be observed in several different parts of the detector, at various off-axis angles, as the roll angle changed between observations.

We expect most of our X-ray sources to be XBs or AGN; furthermore, most of the XBs are likely to be in the "hard state" common to all XBs (van der Klis 1994) at luminosities $\lesssim 10 \%$ Eddington (Gladstone et al. 2007; Tang et al. 2011). The emission spectra of such sources may be estimated by a power law with a photon index $(\Gamma)$ of 1.7 ; hence we initially assumed an absorbed power law model for most X-ray sources, with $\Gamma=1.7$ and line-of-sight absorption $\left(N_{\mathrm{H}}\right)$ equivalent to $7 \times$ $10^{20}$ atom $\mathrm{cm}^{-2}$, the Galactic column density in the direction of M31 (Stark et al. 1992). However, stars and supersoft sources (SSSs) were modeled by $0.05 \mathrm{keV}$ blackbody spectra with $N_{\mathrm{H}}=$ $7 \times 10^{20}$ atom $\mathrm{cm}^{-2}$.

\subsubsection{ACIS}

For ACIS observations, we obtained the response matrices and ancillary response files (ARFs) corresponding to each source spectrum. Net source spectra with $>200$ photons were freely fitted, starting with the assumed spectra, but with $N_{\mathrm{H}}$ and $\Gamma$ free to vary; if the best fit $N_{\mathrm{H}}$ was $<7 \times 10^{20}$ atom cm $^{-2}$, then we fixed $N_{\mathrm{H}}$ to $7 \times 10^{20}$ atom $\mathrm{cm}^{-2}$. If an X-ray source exhibited spectra with $>200$ net counts in only one observation, then we obtained a new lightcurve assuming the best fit model for that observation. If a source had $>200$ source counts in more than one spectrum, then we plotted $N_{\mathrm{H}}$ and $\Gamma$ (or $k T$ ) versus time, and found the best fit line of constant parameter $\left(N_{\mathrm{H}}, \Gamma\right.$ or $\left.k T\right)$. We then converted from intensity to flux using these best fit values for observations with $<200$ net counts.

For transient X-ray sources, we tried two emission models: a power law representing the hard state and a disk blackbody representing the black hole high state that is often observed in black hole transients during the outburst (Remillard \& McClintock 2006). If the disk blackbody model was more successful, then we only applied this model to the outburst. 
We took this approach because it is unfeasible to fit the individual spectra simultaneously, or to create merged spectra for each source. Creating merged spectra is particularly bad because it involves merging data taken from different parts of the detector and at different off-axis angles, making the ancillary response extremely complicated and unreliable. However, simultaneous fitting of the individual observations is also unreliable for sources that vary in luminosity or spectral shape, especially if those observations have poor statistics.

\subsection{2. $H R C$}

For HRC observations, we included only PI channels 48-293, thereby reducing the instrumental background. We used the WebPIMMS tool to find the unabsorbed luminosity equivalent to 1 count $\mathrm{s}^{-1}$ on-axis, assuming the same emission model as for the ACIS observations with $<200$ photons. We created a $1 \mathrm{keV}$ exposure map for each observation and compared the exposure within the source region with that of an equivalent on-axis region in order to estimate the necessary exposure compensation. We multiplied the background subtracted, corrected source intensity by the resulting conversion factor to get the $0.3-10 \mathrm{keV}$ luminosity.

\subsubsection{Creating the Lightcurves}

We created long-term $0.3-10 \mathrm{keV}$ lightcurves for each source, using the luminosities obtained from each observation as described above. We only included observations with net source counts $\geqslant 0$ after background subtraction. We fitted each longterm lightcurve with a line of constant intensity in order to ascertain source variability.

We also made $0.5-4.5 \mathrm{keV}$ flux lightcurves for each source that allowed us to make SFs that were directly comparable with the ensemble AGN SF constructed by Vagnetti et al. (2011). We calculated the $0.5-4.5 \mathrm{keV}$ fluxes using the best-fit emission model where possible; if no spectral fits were made for a source, then we assumed a typical spectrum with $N_{\mathrm{H}}=7 \times$ $10^{20}$ atom $\mathrm{cm}^{-2}$ and a power law with a spectral index of 1.7.

We note that the SF for each source is expected to be rather insensitive to the emission model used to convert from intensity to flux. This conversion is a scaling factor that is applied to the intensity lightcurve after correcting for instrumental effects; hence, the emission model is only important if the parameters significantly change between observations.

\subsection{Characteristics of the Structure Function}

In this work, we compare the SFs of our X-ray sources with the ensemble AGN SF derived from the XMM-Newton data by Vagnetti et al. (2011): $\operatorname{SF}(\tau) \propto \tau^{0.10 \pm 0.01}$. We estimated the normalization of this relation to be 0.11 from Figure 5 of Vagnetti et al. (2011). Therefore the $3 \sigma$ upper limit to the AGN SF derived from the XMM-Newton data is given by $0.11 \tau^{0.13}$.

Vagnetti et al. (2011) calculated the noise component from

$$
\sigma_{n}^{2}=2\left\langle\left(\sigma \log f_{\mathrm{X}}\right)^{2}\right\rangle \simeq 2(\log e)^{2}\left\langle\left(\frac{\sigma f_{\mathrm{X}}}{f_{\mathrm{X}}}\right)^{2}\right\rangle
$$

assuming that $\sigma f_{\mathrm{X}} / f_{\mathrm{X}}=\left(1 / N_{\text {phot }}\right)^{0.5}$, and $N_{\text {phot }}$ is the number of photons. This assumes that all of the noise in the SF comes from Poisson statistics.

Our lightcurves are background subtracted and ARFcorrected; furthermore, uncertainties in the luminosities of bright sources include uncertainties in the spectral parameters. As a result, our uncertainties are not simply due to photon counting noise and may be considerably larger. Hence, in our case,

$$
\sigma_{n}^{2} \simeq(\log e)^{2}\left\langle\left[\frac{\sigma f_{\mathrm{X}}(t+\tau)}{f_{\mathrm{X}}(t+\tau)}\right]^{2}+\left[\frac{\sigma f_{\mathrm{X}}(t)}{f_{\mathrm{X}}(t)}\right]^{2}\right\rangle,
$$

where $\sigma f_{\mathrm{X}}$ is uncertainty in the $\mathrm{X}$-ray flux of a particular observation. The simplest possible SF comes from a single pair of observations with X-ray fluxes $f_{1}$ and $f_{2}$; in this case,

$$
\sigma_{n}^{2} \simeq(\log e)^{2}\left[\left(\frac{\sigma f_{1}}{f_{1}}\right)^{2}+\left(\frac{\sigma f_{2}}{f_{2}}\right)^{2}\right]
$$

A constant X-ray source will yield an SF consistent with zero in all $\tau$ channels. However, if the variation is smaller than the uncertainty in a given channel, then the SF is imaginary for that channel. Our SFs show imaginary channels with zero power but finite uncertainties; channels that contain no observation pairs have zero power and zero uncertainties.

For each source, we calculated the probability, $P_{\mathrm{AGN}}$, that it was consistent with being a typical AGN like the ones studied by Vagnetti et al. (2011). This was derived from $E_{i}$, the excess in channel $i$ over the $3 \sigma$ limit to the AGN SF observed with XMM-Newton $\left(0.11 \tau^{0.13}\right)$; each SF contained $19 \tau$ channels.

$$
P_{\mathrm{AGN}}=\prod_{i=1}^{19} p_{i}
$$

where $p_{i}=1$ when $E_{i} \leqslant 0$, and

$$
p_{i}=1-\frac{2}{\sqrt{\pi}} \int_{0}^{E_{i} / \sqrt{2}} e^{-t^{2}} d t \equiv \operatorname{erfc}\left(E_{i} / \sqrt{2}\right)
$$

when $E_{i}>0$. We then assigned a Rank to each source, given by $-\log \left(P_{\mathrm{AGN}}\right)$. A Rank of 2.6 indicates a $3 \sigma$ excess in $\operatorname{SF}(\tau)$ over $0.11 \tau^{0.13}$, while a Rank of 6.2 indicates a $5 \sigma$ excess.

\section{RESULTS}

\subsection{Overview}

We will first provide an overview of our results before dealing with specific aspects of our data. Table 1 summarizes our main results. For each source, we give its position with respect to the LGS M31 Field $5 B$ band image provided by Massey et al. (2006), along with $1 \sigma$ position uncertainties. Sources marked with "a" have locations derived from XMM-Newton observations and $1 \sigma$ uncertainties calculated from the $3 \sigma$ uncertainties quoted by Stiele et al. (2011). Sources marked with "b" have positions derived from a highly binned image, where one image pixel is equivalent to $9 \times 9$ native pixels. We then show the number of ACIS and HRC observations that contain the source.

Next in Table 1 we present the published source classification and properties. We first show the classification in the Stiele et al. (2011) XMM-Newton catalog of M31 X-ray sources; sources can be classified as XBs (XB), GCs, stars (*), galaxies (Gal), AGN (AGN), supernova remnants (SNR), SSSs, or simply hard $(\mathrm{H})$; square brackets indicate candidates, while exclamation marks indicate classifications that were rejected for our Chandra locations. The remaining X-ray sources either were observed but not classified (NC) or had no entry at all (NE). Any improvement on the Stiele et al. (2011) classification is indicated by "="; 
Table 1

Summary of Our Findings for the 528 X-Ray Sources in Our Survey

\begin{tabular}{|c|c|c|c|c|c|c|c|c|c|}
\hline Src & Pos & $\begin{array}{c}\sigma_{\text {R.A. }} \\
\left({ }^{\prime \prime}\right)\end{array}$ & $\begin{array}{c}\sigma_{\text {Decl. }} \\
\left({ }^{\prime \prime}\right)\end{array}$ & $O_{\mathrm{A}}$ & $O_{\mathrm{H}}$ & Properties & $L_{\mathrm{con}} / 10^{37}$ & $\chi^{2} /$ dof & SF Rank \\
\hline 1 & $00: 41: 05.41+41: 18: 14.7^{\mathrm{a}}$ & $1.3^{\mathrm{a}}$ & $1.3^{\mathrm{a}}$ & 11 & 8 & $\mathrm{H}$ & $0.11 \pm 0.03$ & $13 / 18$ & $7.3^{\mathrm{f}}$ \\
\hline 2 & $00: 41: 05.46+41: 17: 34.1^{\mathrm{a}}$ & $1.0^{\mathrm{a}}$ & $1.0^{\mathrm{a}}$ & 16 & 11 & $\mathrm{H}$ & $0.062 \pm 0.019$ & $22 / 26$ & $0.5^{\mathrm{g}}$ \\
\hline 3 & $00: 41: 07.92+41: 13: 44.3^{\mathrm{a}}$ & $0.8^{\mathrm{a}}$ & $0.8^{\mathrm{a}}$ & 21 & 12 & $\mathrm{H}$ & $0.084 \pm 0.019^{\mathrm{d}}$ & $36 / 32$ & $0.9^{g}$ \\
\hline 4 & $00: 41: 09.15+41: 15: 17.9^{\mathrm{a}}$ & $1.2^{\mathrm{a}}$ & $1.2^{\mathrm{a}}$ & 20 & 17 & $\mathrm{H}$ & $0.063 \pm 0.016$ & $16 / 36$ & $0.2^{\mathrm{g}}$ \\
\hline 5 & $00: 41: 10.73+41: 21: 20.0^{\mathrm{a}}$ & $0.9^{\mathrm{a}}$ & $0.9^{\mathrm{a}}$ & 12 & 20 & {$[*]=*$} & $0.11 \pm 0.02$ & $219 / 31$ & 51.5 \\
\hline 6 & $00: 41: 11.40+41: 20: 05.6^{\mathrm{a}}$ & $0.9^{\mathrm{a}}$ & $0.9^{\mathrm{a}}$ & 13 & 12 & {$[*]$} & $0.09 \pm 0.02^{\mathrm{d}}$ & $8 / 24$ & 0.0 \\
\hline 7 & $00: 41: 11.83+41: 23: 43.6^{\mathrm{a}}$ & $1.4^{\mathrm{a}}$ & $1.4^{\mathrm{a}}$ & 9 & 9 & $\mathrm{H}$ & $0.10 \pm 0.03$ & $10 / 17$ & $0.0^{\mathrm{g}}$ \\
\hline 8 & $00: 41: 12.42+41: 14: 57.6^{\mathrm{a}}$ & $0.8^{\mathrm{a}}$ & $0.8^{\mathrm{a}}$ & 19 & 25 & $\mathrm{H}$ & $0.139 \pm 0.017$ & $59 / 43$ & $2.6^{\mathrm{f}}$ \\
\hline 9 & $00: 41: 13.96+41: 14: 34.5^{\mathrm{a}}$ & $1.2^{\mathrm{a}}$ & $1.2^{\mathrm{a}}$ & 22 & 26 & $\mathrm{H}$ & $0.105 \pm 0.016^{\mathrm{d}}$ & $55 / 47$ & $1.4^{\mathrm{g}}$ \\
\hline 10 & $00: 41: 14.48+41: 14: 00.2^{\mathrm{a}}$ & $1.1^{\mathrm{a}}$ & $1.1^{\mathrm{a}}$ & 20 & 27 & {$[*]$} & $0.081 \pm 0.015$ & $43 / 46$ & 0.5 \\
\hline
\end{tabular}

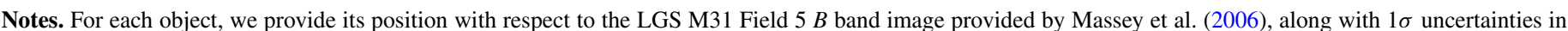

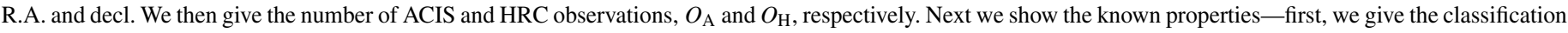

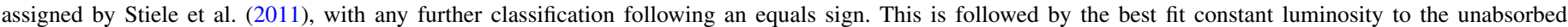
$0.3-10 \mathrm{keV}$ luminosity lightcurve and corresponding $\chi^{2} /$ dof. Finally, we rank the structure function for variability.

${ }^{a}$ Positions and uncertainties obtained from Stiele et al. (2011).

${ }^{b}$ Position uncertainty obtained from a $9 \times 9$ binned image.

${ }^{c}$ HRC observations ignored.

${ }^{\mathrm{d}}$ HRC observations included in luminosity fit but not SF.

e Fitted with blackbody model $N_{\mathrm{H}}=7 \times 10^{20}$ atom $\mathrm{cm}^{-2}, k T=0.05 \mathrm{keV}$.

${ }^{\mathrm{f}}$ Likely XB.

g Consistent with AGN.

(This table is available in its entirety in a machine-readable form in the online journal. A portion is shown here for guidance regarding its form and content.)

these can be known X-ray sources that do not appear in the $X M M-N e w t o n$ catalog $(\mathrm{X}), \mathrm{BHCs}$, transients $(\mathrm{T})$, novae (Nova), ultraluminous X-ray sources (U, exhibiting X-ray luminosities $>2 \times 10^{39}$ erg s$^{-1}$; Kaur et al. 2012; Nooraee et al. 2012; Middleton et al. 2013; Barnard et al. 2013b), or variable stars (var*).

Finally, in Table 1 we show the best-fit constant $0.3-10 \mathrm{keV}$ luminosity over our $\sim 13 \mathrm{yr}$ monitoring program, along with the corresponding $\chi^{2} /$ dof, and our ranking of the object according to the variability shown in its SF. Sources with a Rank $>2.6$ are significantly more variable than expected for typical AGN. For 28 sources indicated with "c ", we ignored the HRC observations completely; for 120 sources indicated with "d," we included HRC observations in the luminosity calculation but exclude $\mathrm{HRC}$ data from the SF; reasons for these decisions are given below. The 10 sources indicated with "e" are very soft and were modeled using an absorbed blackbody with $N_{\mathrm{H}}=7 \times$ $10^{20}$ atom $\mathrm{cm}^{-2}$ and $k T=0.05 \mathrm{keV}$. Using our standard emission model for these sources resulted in large systematic offsets between ACIS and HRC luminosities. Likely XBs are indicated by "f " while "g" indicates sources that are consistent with AGN.

Our luminosities assume a distance of $780 \mathrm{kpc}$ (Stanek \& Garnavich 1998). Clearly the distances to foreground stars and background AGN will be very different, along with their corresponding luminosities. In each case, the flux is given by the luminosity divided by $7.3 \times 10^{49} \mathrm{~cm}^{2}$.

The following sections describe our source list, spectral analysis, and time variability studies in detail.

\subsection{The Source List}

We initially identified 407 X-ray sources from our Chandra observations; registering the merged $0.3-7 \mathrm{keV}$ image to the LGS $B$ band optical image resulted in $1 \sigma$ positional uncertainties of 0'.11 in right ascension (R.A.) and 0'.09 in declination (decl.). Each X-ray source also has an uncertainty in its location on the
X-ray image, which is dependent on the source intensity and off-axis angle.

We compared our X-ray sources with the M31 XMM-Newton source catalog of Stiele et al. (2011) by looking for matches within $10^{\prime \prime}$ of our Chandra positions; these associations were then accepted or rejected based on the $3 \sigma$ uncertainties in the XMM-Newton position reported by Stiele et al. (2011). The XMM-Newton catalog of Stiele et al. (2011) contained $\sim 170 \mathrm{X}$ ray sources that were not in our catalog. We extracted lightcurves and spectra from regions at the positions of these sources and found evidence for a further $121 \mathrm{X}$-ray sources; we found no trace of $\sim 50$ of the X-ray sources in the Stiele et al. (2011). We obtained the positions and uncertainties for the 121 new X-ray sources from Stiele et al. (2011); our total source list contains $528 \mathrm{X}$-ray sources.

We found that 208 of our X-ray sources had no counterpart in the XMM-Newton catalog despite both catalogs having similar luminosity limits $\left(\gtrsim 10^{35} \mathrm{erg} \mathrm{s}^{-1}\right)$. While 36 of these sources are transient, the remainder were likely missed by XMM-Newton because of its larger PSF and higher background. A further 169 X-ray sources were classified only as "hard" in Stiele et al. (2011), while another nine X-ray sources were detected but not classified. Identified sources included 38 GCs or GC candidates; 15 AGN or candidates, including 2 optically identified AGN; 11 SNR or candidates; 28 foreground stars or candidates; 21 SSS candidates; 4 novae or nova candidates; and $28 \mathrm{XBs}$ or $\mathrm{XB}$ candidates, including 13 transients and 5 BHCs (Stiele et al. 2011).

We rejected four AGN, two GC, and three SNRs identified in the Stiele et al. (2011) catalog after obtaining our superior Chandra positions. Our total inventory includes 47 foreground stars or candidates; 7 novae or nova candidates; 12 SNRs or candidates; and 13 SSS candidates with no further classification. Also included are $47 \mathrm{GCs}$ or GC candidates; 37 BHCs (12 in $\mathrm{GCs}$ ), with $1 \mathrm{GC}$ BHC new to this work; and 52 transients (6 located in GCs). 
Table 2

Spectral Fits for Sources with $>200$ Net Source Counts in at Least One ACIS Observation

\begin{tabular}{lccccc}
\hline \hline Src & Model & $N_{\mathrm{H}} / 10^{22}$ & $\chi^{2} /$ dof & Param & $\chi^{2} /$ dof \\
\hline 31 & PO & $0.3 \pm 0.2$ & $\ldots$ & $2.3 \pm 0.9$ & $\ldots$ \\
43 & PO & $0.31 \pm 0.04$ & $4 / 11$ & $2.37 \pm 0.11$ & $3 / 11$ \\
55 & PO & $0.19 \pm 0.12$ & $\ldots$ & $2.1 \pm 0.4$ & $\ldots$ \\
78 & PO & $0.34 \pm 0.04$ & $8 / 9$ & $1.85 \pm 0.07$ & $3 / 9$ \\
83 & PO & $0.33 \pm 0.14$ & $2 / 3$ & $1.57 \pm 0.18$ & $1.1 / 3$ \\
84 & PO & $0.50 \pm 0.13$ & $1.6 / 5$ & $2.3 \pm 0.2$ & $1.4 / 5$ \\
90 & PO & $0.11 \pm 0.07$ & $0.13 / 2$ & $1.7 \pm 0.2$ & $0.31 / 2$ \\
95 & PO & $0.45 \pm 0.09$ & $0.2 / 3$ & $1.85 \pm 0.13$ & $1.4 / 3$ \\
100 & BB & $0.129 \pm 0.019$ & $12 / 47$ & $0.572 \pm 0.007$ & $37 / 47$ \\
103 & PO & $0.28 \pm 0.06$ & $0.13 / 4$ & $2.12 \pm 0.13$ & $4 / 4$ \\
106 & PO & $0.12 \pm 0.12$ & $\ldots$ & $1.7 \pm 0.4$ & $\ldots$ \\
\hline
\end{tabular}

(This table is available in its entirety in a machine-readable form in the online journal. A portion is shown here for guidance regarding its form and content.)

We estimated the probable number of false associations by finding the number of matches between our X-ray sources and astronomical objects after adding $20^{\prime \prime}$ to or subtracting $20^{\prime \prime}$ from the R.A. or decl. In each of the four tests we found 6-11 false matches with stars, 0-1 false matches with a candidate SNR, 0-2 false matches with candidate GCs, and 1-4 false matches with novae.

\subsection{Spectral Fitting}

We found 120 sources that had spectra with $>200$ net source counts in at least one ACIS observation. We fitted the spectra with the emission model that suited the source classification; stars were fitted with blackbody models, X-ray transients in outburst were fitted with disk blackbody models (see, e.g., Barnard et al. 2012a), and the remaining sources were fitted with power-law models; each model included lineof-sight absorption, with a minimum value equivalent to $7 \times$ $10^{20} \mathrm{H}$ atom $\mathrm{cm}^{-2}$.

High-quality XMM-Newton observations of the brightest sources in our survey required two-component models (e.g., blackbody + power law; see Barnard et al. 2006 for an example); however, Chandra spectra from these sources were acceptably fitted by simple power-laws $(\Gamma \lesssim 1)$. Due to the difference between HRC and ACIS responses, we ignored the HRC data for these systems; even if we used two component emission models, it would be impossible to estimate the HRC luminosities due to the uncertainties in the contributions of the different components.

For sources with multiple spectral fits, we obtained the bestfit constant values for the absorption and emission parameters, along with the corresponding $\chi^{2} /$ dof, where the degrees of freedom (dof) is one less than the number of fitted observations. Table 2 summarizes our findings. For each source we provide the emission model used (power law "PO," blackbody "BB," or disk blackbody "DBB”), absorption $\left(N_{\mathrm{H}} / 10^{22}\right.$ atom $\left.\mathrm{cm}^{-2}\right)$ plus $\chi^{2} /$ dof, and parameter (photon index for $\mathrm{PO}, k T / \mathrm{keV}$ for $\mathrm{BB}$ or DBB) plus $\chi^{2} /$ dof. If only one observation was fitted, then the $\chi^{2} /$ dof is given as ..." in cases with only two observations ( 1 dof $), \chi^{2}$ was often extremely low and meaningless. The absorption was fixed to Galactic line of sight absorption $(7 \times$ $10^{20}$ atom $\mathrm{cm}^{-2}$ ) for three sources (S175, S250, and S287).

In many cases, the parameter fits have low $\chi^{2} /$ dof; this is to be expected when each datum is itself derived from fitting a spectrum that may have large uncertainties. Only one source
(S327) exhibited significant variation in absorption. Five sources (S117, S209, S251, S327, and S396) exhibited significant variability in their emission parameters; indeed the variability in S209 was so extreme that the HRC observations were unusable (each ACIS observation had sufficient counts for fitting). S117, S251, and S396 are transients; S209 is a candidate Z-source (Barnard et al. 2003); and S327 is a BHC (Barnard et al. 2013a).

It is unsurprising that so few X-ray sources exhibited spectral variability, since the hard state is observed up to $\sim 0.1$ times the Eddington limit $\left(\sim 2 \times 10^{37} \mathrm{erg} \mathrm{s}^{-1}\right.$ for a $1.4 M_{\odot}$ neutron star (NS)), and $\sim 90 \%$ of our sample is consistent with being in the NS hard state. For example, S213 has a best-fit luminosity $\sim 10^{37} \mathrm{erg} \mathrm{s}^{-1}$ and a Rank $>320$, and the entire $0.3-10 \mathrm{keV}$ lightcurve is consistent with S213 being in an NS hard state. Furthermore, the relatively poor statistics for these distant sources led to relatively large uncertainties in the emission parameters, making significant variability harder to detect than for Galactic XBs.

\subsection{X-Ray Identification of Foreground Stars}

Many foreground stars, or candidates, were identified by their proximity to an optical counterpart. Some of these stars were identified as "hard" by Stiele et al. (2011) due to overlapping selection criteria (HR2-EHR2 $>-0.2$ for hard sources with no other classification, but HR2-EHR2 $<0.3$ for foreground stars along with other criteria). We label these sources as " $\mathrm{H}=[*]$."

It was therefore necessary to investigate these sources further using the best ACIS spectra available; if the observed counts fell mostly below $1 \mathrm{keV}$, then we considered the source a foreground star, indicated by " $\mathrm{H}=[*], *$ ". Otherwise we considered them "hard" sources, " $\mathrm{H}=[*], \mathrm{H}$ ". If there were too few counts to even determine this much, then we conservatively classify the source as a candidate star, "H=[*],[*]". Two sources (S34, and S74) were classified as stars, and four sources (S138, S165, S282, S402) were classified as candidate stars. Seven sources (S87, S92, S340, S399, S405, S499, and S507) were classified as hard.

\subsection{Long-term Variability}

We assessed the long-term variability of each X-ray source in two ways: the $0.3-10 \mathrm{keV}$ lightcurves and the $0.5-4.5 \mathrm{keV}$ SFs. The instrumental responses of the ACIS and HRC instruments are significantly different, and only the ACIS observations are capable of providing reliable spectral data. Therefore, we made two lightcurves and two SFs for each source, one including ACIS and HRC observations and one including only ACIS data.

There are two situations in which the inclusion of HRC data may be detrimental to our studies of a particular X-ray source. In the first situation, where the HRC lightcurve is systematically offset from the ACIS lightcurve due to differences in instrumental response, we exclude the HRC observations entirely; such cases are indicated by "c" in Table 1 . In the second situation, where particularly large uncertainties in HRC observations dominate the SFs, we include the HRC observations in our luminosity estimates, but exclude them from our SFs. These cases are indicated by "d" in Table 1 . Whenever the two SFs for a particular source gave conflicting results, we favored the ACIS-only SF.

The first situation can affect any source, but it is particularly important for bright sources exhibiting significant spectral evolution. The ACIS and HRC luminosities only agree if the chosen emission model is appropriate for the observed spectrum. For 

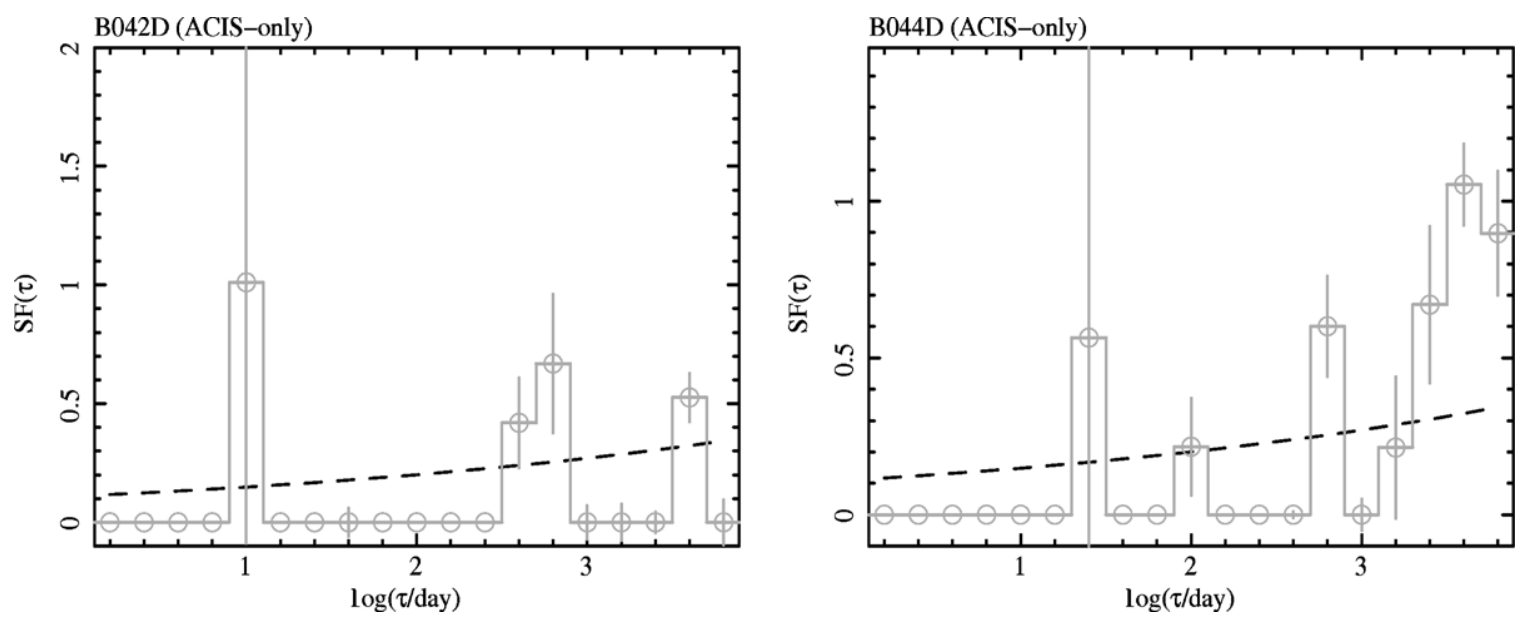

Figure 1. $0.5-4.5 \mathrm{keV}$ ACIS-only SFs for the two AGN associated with optical galaxies, B042D and B044D. The dashed curves represent $\mathrm{SF}(\tau)=0.11 \tau^{0.13}$, the $3 \sigma$ upper limit to the ensemble AGN SF created by Vagnetti et al. (2011) from XMM-Newton data. These galaxies have luminosities $\sim 10^{42}$ and $\sim 10^{41} \mathrm{erg} \mathrm{s}^{-1}$ respectively, 1-5 orders of magnitude lower than the galaxies in the sample used by Vagnetti et al. (2011).

most sources, we must assume a standard spectrum. This is successful in many cases, but for those that are unsuccessful, using only the ACIS data minimizes the uncertainties in luminosity and variability.

The second situation affects some faint X-ray sources. Some HRC observations have exposure times as short as $1 \mathrm{ks}$; since the sensitivity of the HRC is a factor $\sim 5$ lower than the ACIS for typical XB spectra, the large uncertainties in HRC luminosities can dominate the SF to the extent that variability between ACIS observations is lost.

The $0.5-10 \mathrm{keV}$ flux distribution obtained by Georgakakis et al. (2008) leads us to expect 635 AGN within our 20' region with fluxes $\gtrsim 1.4 \times 10^{-15} \mathrm{erg} \mathrm{cm}^{-2} \mathrm{~s}^{-1}$, equivalent to luminosities $>10^{35} \mathrm{erg} \mathrm{s}^{-1}$ at $780 \mathrm{kpc}$. If the sensitivity of our survey were uniform, we would expect to observe $<2$ typical AGN (as defined by Vagnetti et al. 2011) with a Rank of $\geqslant 2$.6. Considering the deterioration of sensitivity with off-axis angle and the fact that our uncertainties in the backgroundsubtracted luminosities are often substantially larger than the Poisson statistics assumed by Vagnetti et al. (2011), we expect $\ll 1$ typical AGN to exhibit Rank $\geqslant 2.6$.

Since we assume a particular emission model to convert from counts to luminosity in many cases, spectral variation in the $\mathrm{X}$-ray sources would lead to systematic uncertainties. However, $\sim 90 \%$ of our sample exhibited $0.3-10 \mathrm{keV}$ luminosities consistent with the NS hard state throughout our $13 \mathrm{yr}$ monitoring campaign, hence we expect little spectral variation from these sources. Furthermore, only 5 out of the 120 X-ray sources with freely fitted spectra exhibited significant spectral variation. Hence spectral change is unlikely to be a major source of systematic uncertainties in estimating luminosity variability.

\subsubsection{Classifying the X-Ray Sources}

We found 246 sources with an SF Rank of $\geqslant 2.6$, and 282 sources with a Rank of $<2.6$. Sources with a Rank of $\geqslant 2.6$ include 18 foreground stars, 5 SSSs, 7 SNRs, 1 nova, and 2 low-luminosity AGN (discussed below); the SNRs are extended and not expected to be variable. Hence we assume that the associations of strongly variable X-ray sources with SNRs are coincidental. Sources with a Rank of $<2.6$ include 11 transients, 13 sources associated with GCs, 29 stars, 8 SSSs, 6 novae, and 6 bright XBs.
We classify as XBs all 52 X-ray transients (this number excludes novae), all $47 \mathrm{GC}$ sources, the 7 variable sources associated with SNRs, 144 unclassified X-ray sources with Ranks of $\geqslant 2.6$, and 6 bright XBs with Ranks of $<2.6$; we therefore identify $250 \mathrm{XBs}$ in total (6 transients are located in GCs). We do not count the novae or unidentified SSSs, although some of them may be XBs. It is perhaps surprising that 11 transients exhibited Ranks of $<2.6$; however, the lightcurves for these sources include many observations when they were in quiescence, meaning that the noise dominates the SFs for these sources. We found $161 \mathrm{XBs}$ with Ranks of $\geqslant 6.2$ (a $5 \sigma$ rejection of the AGN classification), and 36 XBs have Ranks of $>320$, which the limit imposed by machine precision.

We found that 202 unclassified X-ray sources and one galaxy candidate identified by Stiele et al. (2011) exhibited Ranks of $<2.6$ and are consistent with being AGN. In addition there are two optically identified AGN that exhibit high variability: the ACIS-only SF for S73 has a Rank of 2.6, while the ACISonly SF for S75 has a Rank of 12.3 (shown in Figure 1). The ACIS+HRC SFs were dominated by noise from the HRC. However, these galaxies (B042D and B044D) are relatively nearby (with velocities of $57,833 \mathrm{~km} \mathrm{~s}^{-1}$ and $35,743 \mathrm{~km} \mathrm{~s}^{-1}$, respectively; Caldwell et al. 2009), and exhibited low luminosities $\left(\sim 10^{42} \mathrm{erg} \mathrm{s}^{-1}\right.$ and $\left.\sim 10^{41} \mathrm{erg} \mathrm{s}^{-1}\right)$. The ensemble AGN SF created by Vagnetti et al. (2011) sampled AGN with luminosities $10^{43}-10^{45.5}$ erg s$^{-1}, \sim 1-5$ orders of magnitude higher than S73 and S75. Therefore we estimated the variability seen at $10^{41}$ and $10^{42} \mathrm{erg} \mathrm{s}^{-1}$ using the published relations between luminosity and variability: $I_{\mathrm{var}} \propto L^{-0.21}$ over $\sim 100$ day timescales (Vagnetti et al. 2011), and $I_{\mathrm{var}} \propto L^{-0.13}$ over year timescales (Markowitz \& Edelson 2004). We found that S73 and S75 were both consistent with the expected AGN SF when scaled to their luminosities. These results do not jeopardize our ranking system, as we expect that other low-luminosity AGN would have to be local to be detected and that such nearby AGN would be identified in other wavelengths.

Figure 2 compares the long-term lightcurves and SFs of three $\mathrm{X}$-ray sources. Panels (a) and (b) show the lightcurve and SF for S381, one of the faintest X-ray sources with a Rank of >2.6. S381 has a mean 0.3-10 keV luminosity of $1.1 \pm 0.3 \times$ $10^{35} \mathrm{erg} \mathrm{s}^{-1}$, with $\chi^{2} /$ dof $=47 / 49$; its SF yielded a Rank of 11.1. We note that the SF includes several bins with zero SF but finite uncertainties; $\operatorname{SF}(\tau)$ is imaginary for these bins, because 

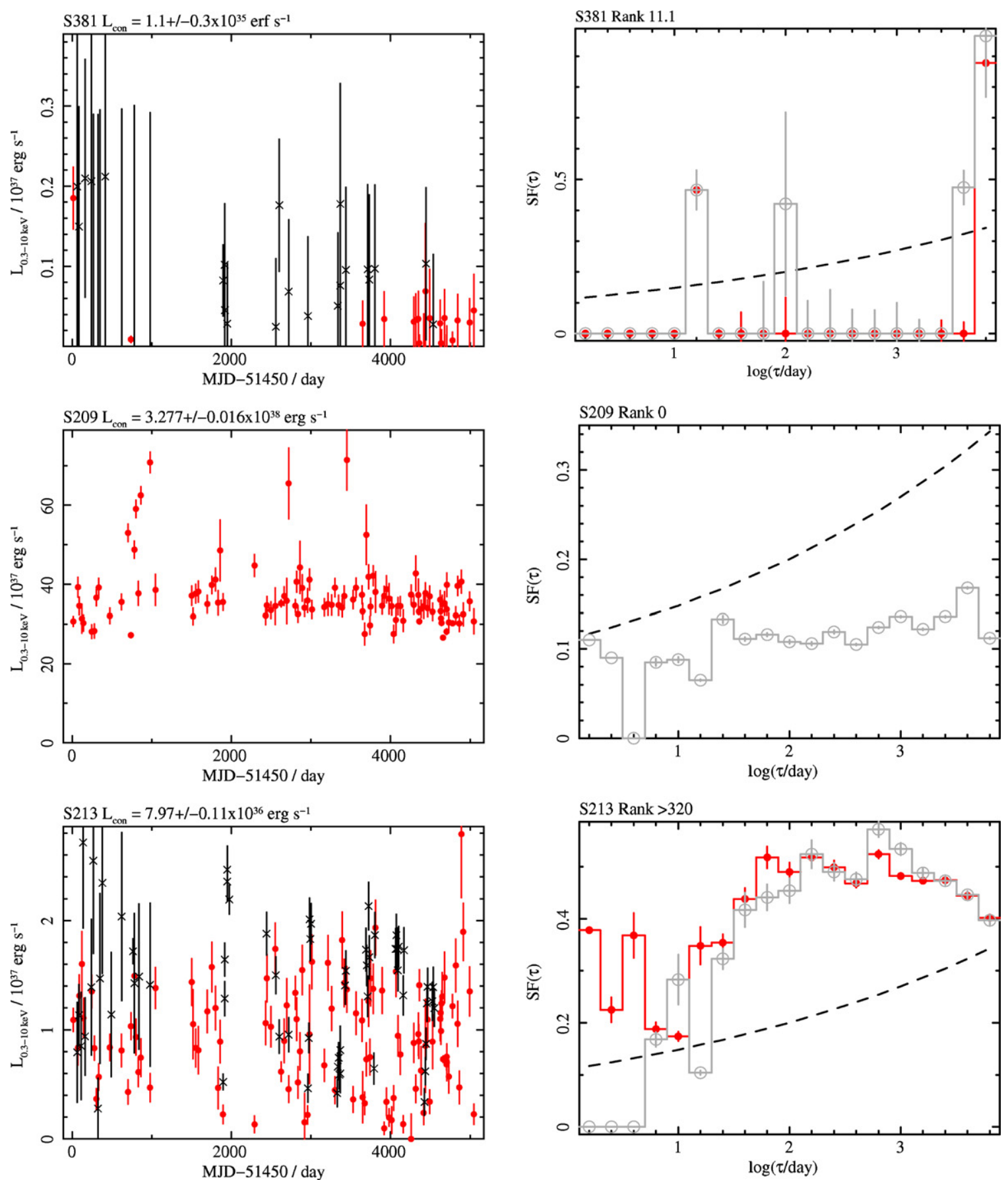

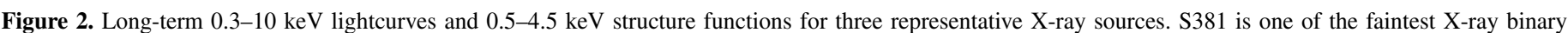

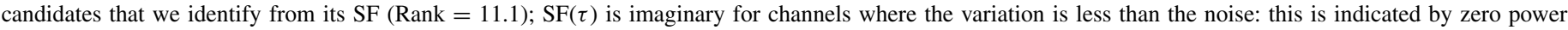

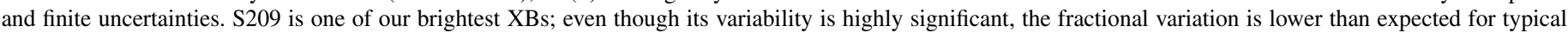

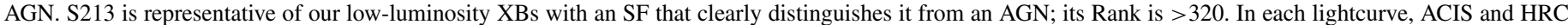

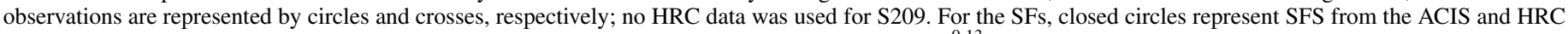
observations, while open circles represent the ACIS-only SFs; dashed lines represent $\operatorname{SF}(\tau)=0.11 \tau^{0.13}$.

(A color version of this figure is available in the online journal.)

the variation is smaller than the noise. Panels (c) and (d) give the ACIS-only lightcurve and SF for S209, one of the brightest $\mathrm{X}$-ray sources in the field of view; $\chi^{2} /$ dof $=1255 / 106$ for its best fit constant intensity, yet its SF shows less fractional variation than a typical AGN over all timescales. S209 is one of those sources where the HRC data were excluded entirely from the analysis. Panels (e) and (f) show the lightcurve and SF for S213, one of the 36 XBs with a Rank of $>320$. These results agree with the well-known behavior of galactic XBs where the high luminosity XBs only vary by a factor of a few, while low luminosity XBs can vary in intensity by $1-2$ orders of magnitude (see, e.g., Muno et al. 2002).

In Figure 3 we compare the Rank and luminosity for our sources identified as XBs and possible AGN. We see that sources with Rank $<2.6$ are found at $0.3-10 \mathrm{keV}$ luminosities $\lesssim 6 \times 10^{36} \mathrm{erg} \mathrm{s}^{-1}$ and $\gtrsim 5 \times 10^{37} \mathrm{erg} \mathrm{s}^{-1}$; it is well known that high luminosity XBs in our Galaxy are substantially less variable than low luminosity XBs (Muno et al. 2002). It is possible that 


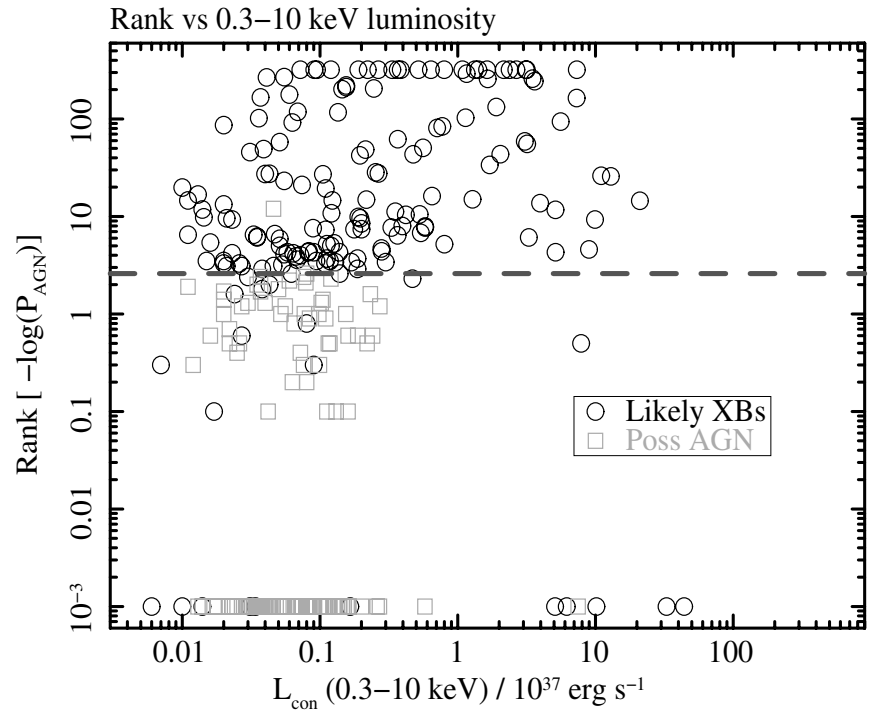

Figure 3. Comparison of Rank (i.e., $-\log \left[P_{\mathrm{AGN}}\right]$ ) vs. $0.3-10 \mathrm{keV}$ luminosity for our X-ray binaries (open circles) and sources consistent with AGN (open squares). The dashed line indicates a Rank of 2.6 , corresponding to a $3 \sigma$ rejection of an AGN identification. An offset of 0.001 was added to those sources with a Rank of 0 to allow them to show up on a log scale.

the intrinsic variability of some faint XBs was obscured by the scarcity of observed photons. The median Ranks for our XBs, sources consistent with $\mathrm{AGN}$, and the remaining sources are $11.2,0.0$, and 1.0 , respectively. Clearly our XB population is vastly more variable than the other classes of sources.

\subsubsection{Comparing Our Possible AGN Population with Expected AGN}

We found 205 X-ray sources to be consistent with AGN; however, this sample may contain some faint XBs. Georgakakis et al. (2008) have created a flux distribution (i.e., $\log N$ versus $\log S$ ) for AGN in the $0.5-10 \mathrm{keV}$ band, which is similar enough to our $0.3-10 \mathrm{keV}$ band for comparison; the $0.3-10 \mathrm{keV}$ and $0.5-10 \mathrm{keV}$ fluxes for our possible AGN agree to within $\sim 10 \%$. If we simply scale this distribution to a circular area with $20^{\prime}$ radius, then we expect 635 AGN with fluxes $>1.4 \times$ $10^{-15} \mathrm{erg} \mathrm{cm}^{-2}$ (equivalent to luminosities $>10^{35} \mathrm{erg} \mathrm{s}^{-1}$ for sources in M31). This is a factor $\sim 3$ times greater than the number of observed AGN candidates and more than the total of observed X-ray sources. The origin of this discrepancy could be astronomical, if absorbing material in the M31 bulge reduces the observed number of faint AGN. Alternatively, this effect could be instrumental, since the sensitivity of the Chandra images is reduced at large off-axis angles by vignetting and reduced exposure. Our normalization of the AGN flux distribution assumes uniform sensitivity.

Therefore, we compared the AGN candidates with the expected AGN within $6^{\prime}$ of the M31 nucleus. We expected 56 AGN and observed 66 AGN candidates within this region. Given that we see an excess of sources consistent with AGN over the expected amount within $6^{\prime}$ of M31*, and only $\sim 30 \%$ expected sources within $20^{\prime}$ of $\mathrm{M} 31^{*}$, this deficit is probably dominated by instrumental effects.

We compare the $0.3-10 \mathrm{keV}$ flux distribution of the observed AGN candidates with the expected $0.5-10 \mathrm{keV}$ flux distribution found by Georgakakis et al. (2008) for the two regions (with radii $20^{\prime}$ and $6^{\prime}$ ) in Figure 4. Solid, stepped lines indicate the observed flux distributions, while dashed lines show the expected distributions. Black lines correspond to the $20^{\prime}$ region,

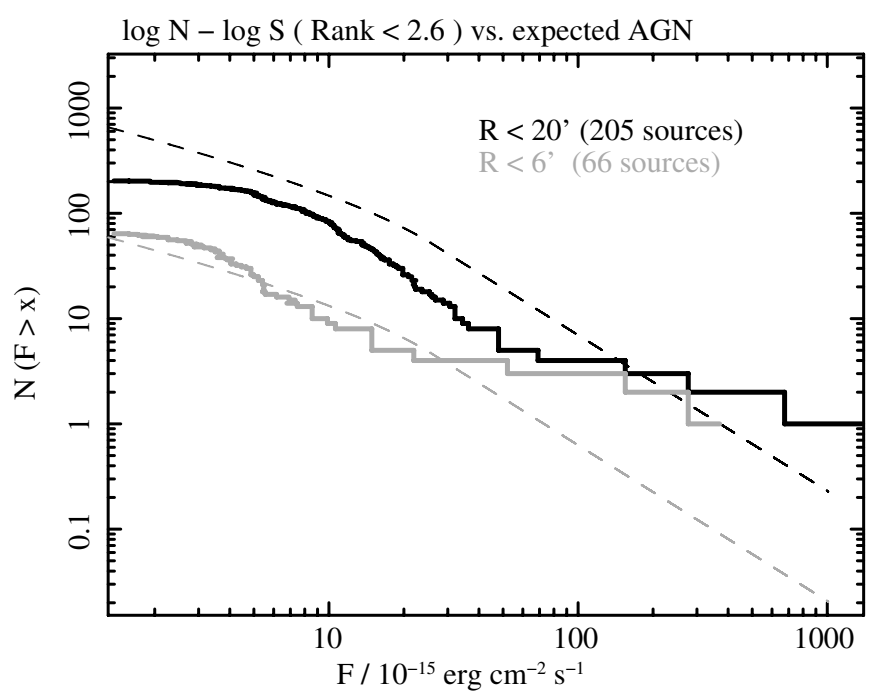

Figure 4. Comparison between the $0.3-10 \mathrm{keV}$ flux distribution of observed AGN candidates (solid lines) and the expected 0.5-10 keV AGN distribution from Georgakakis et al. (2008) (dashed lines). The $0.3-10 \mathrm{keV}$ and $0.5-10 \mathrm{keV}$ fluxes agree to within $10 \%$. Black and gray lines correspond to circular regions with $20^{\prime}$ and $6^{\prime}$ radius, respectively.

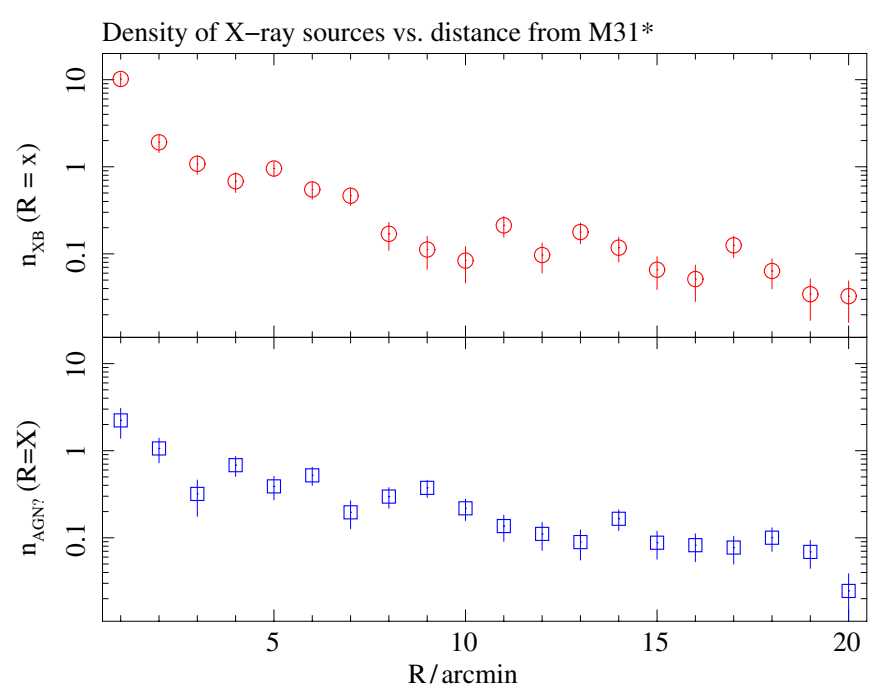

Figure 5. Number densities of our XBs (top) and AGN (bottom) vs. distance from galactic center (in the plane of the detector). We expect $\mathrm{XB}$ density to follow stellar mass, while AGN density is expected to be constant.

(A color version of this figure is available in the online journal.)

and gray lines to the $6^{\prime}$ region. We see that the flux distributions of possible AGN are considerably flatter than expected. Together with the excess seen in the population within $6^{\prime}$, these results suggest that some of the sources with Ranks of $<2.6$ are unidentified XBs.

\subsection{Number Density of XBs and AGN versus Distance from $M 31$ *}

We calculated the number densities of our $250 \mathrm{XBs}$ and our 203 possible AGN per arcmin ${ }^{2}$ versus distance from M31* in the detector plane. We present our findings in Figure 5.

Our variability survey makes no distinction between LMXBs and high mass X-ray binaries (HMXBs). LMXBs are old, and their numbers tend to follow stellar mass; HMXBs are young, and their populations depend on the star formation rate (see, 


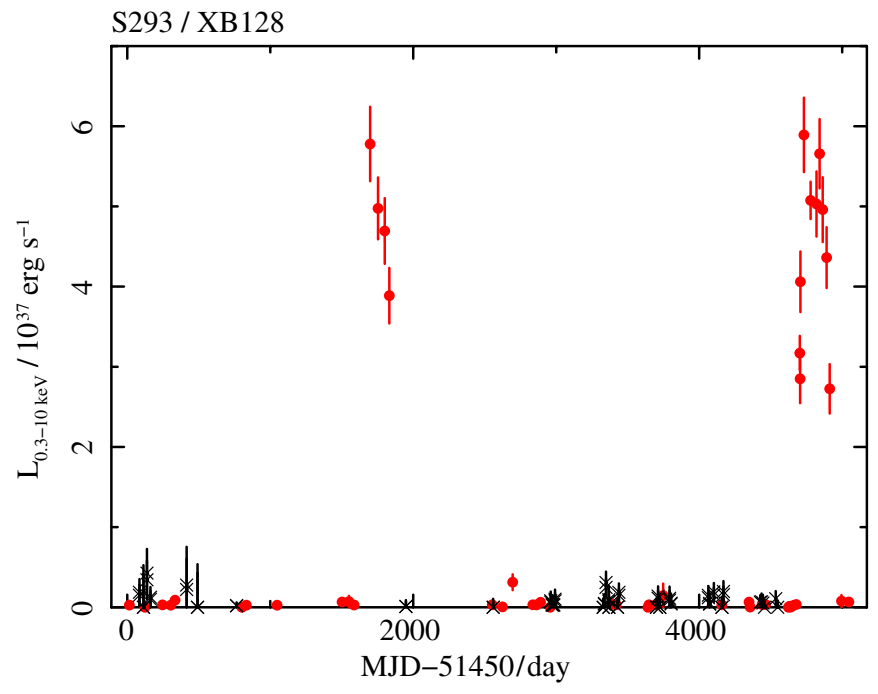

Figure 6. Our long-term 0.3-10 keV luminosity lightcurve for S293/XB128, a recurrent transient X-ray binary associated with the M31 GC B128 that may contain a black hole. ACIS and HRC observations are represented by circles and crosses, respectively.

(A color version of this figure is available in the online journal.)

e.g., Grimm et al. 2003). A proper comparison between the distribution of our XBs and the expected distribution is beyond the scope of this article. However, the observed distribution does appear to agree with expectations (cf. Figure 9 of Voss \& Gilfanov 2007, taking into account differences in normalization due to different luminosity limits).

We might expect the AGN density to remain constant, with a possible decrease at large distances to account for decreasing instrument sensitivity. However, the number density of possible AGN is considerably higher for regions closer to M31*, again consistent with the presence of some XBs in our sample of possible AGN.

\subsection{A Newly Identified Transient Black Hole Candidate in the Globular Cluster B128}

In Barnard et al. (2013a), we identified 26 new BHCs in our Chandra observations using a three-step process. First, we established that the X-ray source was an XB, using SF analysis or by associations with a GC or by flux. Next, we looked for high quality hard-state spectra (represented by power-law emission with a photon index of $\Gamma \sim 1.4-2.1)$ at luminosities above the threshold for NS XBs $\left(\sim 3 \times 10^{37} \mathrm{erg} \mathrm{s}^{-1}\right)$. Finally, we modeled these spectra with disk blackbody + blackbody emission models, and compared the values with the parameter space defined by soft NS XB spectra (Lin et al. 2007, 2009, 2012).

S293 in our survey is a newly identified BHC associated with the confirmed old M31 GC known as B128, following the Revised Bologna Catalog v 3.4 (Galleti et al. 2004, 2006, 2007, 2009). S293, also known as XB128 following our naming convention for X-ray sources associated with GCs (see, e.g., Barnard et al. 2013a), is a transient X-ray source that has exhibited two outbursts during our monitoring campaign. We present a $0.3-10 \mathrm{keV}$ lightcurve of XB128 in Figure 6.

The first outburst occurred in 2004 May, reaching a $0.3-10 \mathrm{keV}$ luminosity of $5.3 \pm 0.4 \times 10^{37} \mathrm{erg} \mathrm{s}^{-1}$ and lasted at least 134 days; unfortunately, the nearest observation containing XB128 prior to the burst was 113 days earlier, and the next one was 114 days after the last bright observation. The first outburst was only seen in decline.
We caught the second outburst during the rise in 2012 August, with a maximum $0.3-10 \mathrm{keV}$ luminosity of $5.9 \pm 0.4 \times$ $10^{37} \mathrm{erg} \mathrm{s}^{-1}$; the true peak may not have been observed. It was still active 210 days after our first detection of the outburst, but had disappeared the next time that region was observed 62 days later.

During a $\sim 40 \mathrm{ks}$ ACIS-S observation (Obs ID 14196), a power-law fit to the spectrum of XB128 gave $N_{\mathrm{H}}=8 \pm 5 \times$ $10^{20}$ atom $\mathrm{cm}^{-2}$ and $\Gamma=1.54 \pm 0.09$ with $\chi^{2} /$ dof $=56 / 63$; the $0.3-10 \mathrm{keV}$ luminosity was $5.1 \pm 0.2 \times 10^{37} \mathrm{erg} \mathrm{s}^{-1}$. This is the best spectrum we have for XB128 that supports a BHC classification, with 1550 net source counts.

We fitted this spectrum with a disk blackbody + blackbody emission model and found the $2-10 \mathrm{keV}$ luminosities for each component. This is for comparison with the NS XB soft-state parameter space that was ascertained from hundreds of $R X T E$ spectra analyzed by Lin et al. (2007, 2009, 2012).

Our criteria for identifying BHCs from their disk blackbody + blackbody fits are presented in Barnard et al. (2013a) and summarized as follows. Lin et al. $(2007,2009)$ were unable to obtain successful fits to hard-state spectra with their double thermal (disk blackbody + blackbody) model; the disk blackbody temperature is forced to low values because it must account for the low energy flux, and it contributes little to the $2-10 \mathrm{keV}$ flux. By contrast, Lin et al. $(2007,2009,2012)$ found the disk blackbody component dominated their bright NS spectra, with temperatures $\gtrsim 1 \mathrm{keV}$. Since we identify our BHCs from high luminosity hard states, we expect double thermal fits to $\mathrm{BHC}$ spectra to result in low temperatures and small contributions to the $2-10 \mathrm{keV}$ flux from the disk blackbody components (Barnard et al. 2013a).

When fitting the double thermal model, the absorption was fixed to $7 \times 10^{20}$ atom $\mathrm{cm}^{-2}$, equivalent to the galactic line-of-sight absorption; the absorption was unreasonably low when freely fitted. The disk blackbody contributed $4 \pm 2 \times$ $10^{36} \mathrm{erg} \mathrm{s}^{-1}$ to the $2-10 \mathrm{keV}$ luminosity, with an inner disk temperature of $0.59_{-0.11}^{+0.16} \mathrm{keV}$. The blackbody component had a temperature of $1.35_{-0.18}^{+0.4} \mathrm{keV}$, emitting $2.4 \pm 0.2 \times 10^{37} \mathrm{erg} \mathrm{s}^{-1}$. The total 2-10 keV luminosity was $2.8 \pm 0.3 \times 10^{37} \mathrm{erg} \mathrm{s}^{-1}$, and $\chi^{2} /$ dof $=57 / 62$. We present the unfolded spectrum fitted with this model in Figure 7 . The disk blackbody temperature was $2.8 \sigma$ below $1 \mathrm{keV}$; furthermore, its $2-10 \mathrm{keV}$ contribution was $3.8 \sigma$ below $50 \%$. Hence, XB128 is unlikely to contain a NS primary (probability of disk blackbody temperature and contribution being consistent with a NS $\sim 7 \times 10^{-7}$ ) and is a strong BHC.

Optical spectroscopy of the GC B128 revealed that it has an age of $\sim 10^{10.1} \mathrm{yr}$, a mass of $\sim 10^{5.32} M_{\odot}$, and metallicity $[\mathrm{Fe} / \mathrm{H}]=-0.56$; it is ranked 68 out of 379 GCs by metallicity $(>82 \%)$ and ranked 204 (>46\%) by mass (Caldwell et al. 2009, 2011). We have previously found 11 GC BHCs, and they are all found in GCs that are significantly more massive, more metal rich, or both, than the general GC population in M31 (Barnard et al. 2008, 2011, 2012b, 2013a; Barnard \& Kolb 2009). The GC B128 continues this trend, being near average mass but particularly metal rich.

\section{SUMMARY AND CONCLUSIONS}

We have applied a novel technique for distinguishing between $\mathrm{XBs}$ and AGN to $528 \mathrm{X}$-ray sources in the central region of M31, observed $\sim 170$ times over the last 13 yr with Chandra. We identified 250 likely XBs in total; this includes $\sim 200$ new 


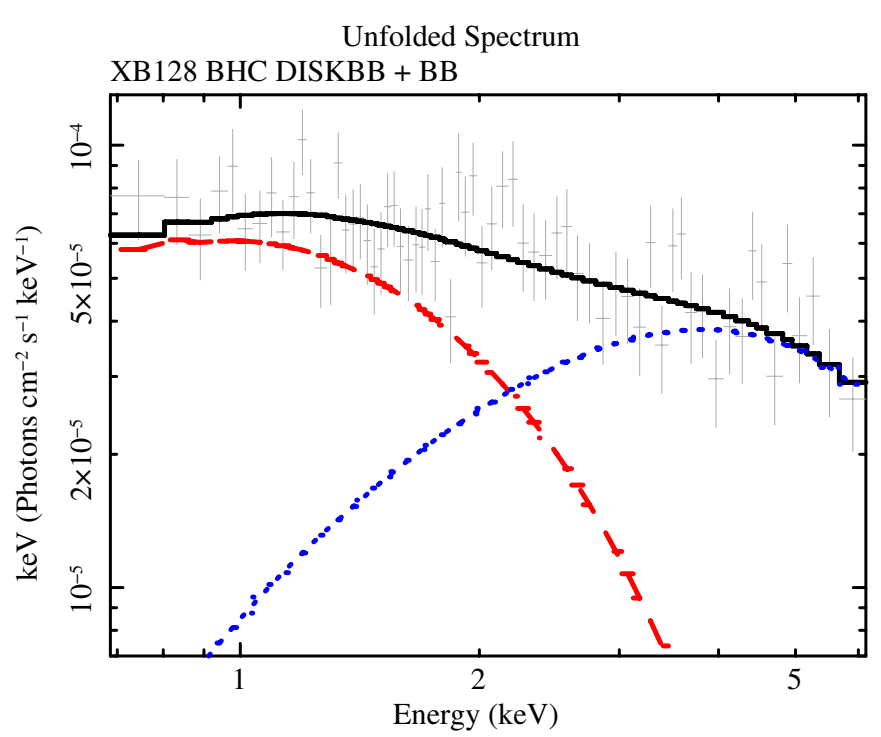

Figure 7. Unfolded spectrum for S293/XB128 from the $\sim 40 \mathrm{ks}$ ACIS-S observation 14196, with the best fit double thermal model. The disk blackbody and blackbody components are represented by dashed and dotted curves, respectively. The $y$-axis is multiplied by channel energy.

(A color version of this figure is available in the online journal.)

$\mathrm{XB}$ identifications. Previously, only $\sim 50 \mathrm{XBs}$ and $\sim 40 \mathrm{XB}$ candidates had been identified out of $\sim 2000$ X-ray sources within the $D_{25}$ region of M31 (Stiele et al. 2011).

We find that up to $205 \mathrm{X}$-ray sources with $0.3-10 \mathrm{keV}$ fluxes $\gtrsim 1.4 \times 10^{-15} \mathrm{erg} \mathrm{cm} \mathrm{cm}^{-2} \mathrm{~s}^{-1}$ are consistent with being AGN; this is a factor $\sim 3$ lower than expected from the $0.5-10 \mathrm{keV}$ flux distribution found by Georgakakis et al. (2008). We find that this difference is due to instrumental effects: simply scaling the Georgakakis et al. (2008) relation by area implies a constant sensitivity, but the Chandra sensitivity significantly decreases with increasing off-axis angle.

Our SF analysis technique for identifying XBs could be applied to several well-studied nearby galaxies. We find that the low luminosity XBs tend to be significantly more variable than the high luminosity XBs (in agreement with observations of Galactic XB behavior; Muno et al. 2002); hence this technique is not suitable for distant galaxies where only very luminous XBs are detected.

Furthermore, we identify a new transient BHC, associated with the confirmed GC B128. This X-ray source, known as XB128, exhibited two outbursts during our monitoring observations. We obtained a $40 \mathrm{ks}$ ACIS-S spectrum during the observed peak of the second outburst and fitted it with a disk blackbody + blackbody emission model for comparison with the full gamut of spectra exhibited by Galactic NS binaries and analyzed by Lin et al. (2007, 2009, 2012). The disk blackbody component for XB128 was inconsistent with NS spectra at a $>5 \sigma$ level, hence we label it a BHC, following Barnard et al. (2013a).
We thank the anonymous referee, whose thoughtful comments substantially improved this article. This research has made use of data obtained from the Chandra data archive and software provided by the Chandra X-ray Center (CXC). This work was supported by Chandra grants GO2-13106X, and GO314095X.

\section{Facilities: CXO (ACIS), CXO (HRC)}

\section{REFERENCES}

Barnard, R., Church, M. J., \& Bałucińska-Church, M. 2003, A\&A, 405, 237

Barnard, R., Foulkes, S. B., Haswell, C. A., et al. 2006, MNRAS, 366, 287

Barnard, R., Galache, J. L., Garcia, M. R., et al. 2012a, ApJ, 756,32

Barnard, R., Garcia, M., \& Murray, S. S. 2012b, ApJ , 757, 40

Barnard, R., Garcia, M. R., \& Murray, S. S. 2011, ApJ, 743, 185

Barnard, R., Garcia, M. R., \& Murray, S. S. 2013a, ApJ, 770, 148

Barnard, R., Garcia, M. R., Murray, S. S., \& Primini, F. 2013b, ApJ, 772,126

Barnard, R., \& Kolb, U. 2009, MNRAS, 397, L92

Barnard, R., Stiele, H., Hatzidimitriou, D., et al. 2008, ApJ, 689, 1215

Caldwell, N., Harding, P., Morrison, H., et al. 2009, AJ, 137, 94

Caldwell, N., Schiavon, R., Morrison, H., Rose, J. A., \& Harding, P. 2011, AJ, 141,61

Galleti, S., Bellazzini, M., Buzzoni, A., Federici, L., \& Fusi Pecci, F. 2009, A\&A, 508, 1285

Galleti, S., Bellazzini, M., Federici, L., Buzzoni, A., \& Fusi Pecci, F. 2007, A\&A, 471,127

Galleti, S., Federici, L., Bellazzini, M., Buzzoni, A., \& Fusi Pecci, F. 2006, A\&A, 456, 985

Galleti, S., Federici, L., Bellazzini, M., Fusi Pecci, F., \& Macrina, S. 2004, A\&A, 416,917

Georgakakis, A., Nandra, K., Laird, E. S., Aird, J., \& Trichas, M. 2008, MNRAS, 388,1205

Gladstone, J., Done, C., \& Gierliński, M. 2007, MNRAS, 378, 13

Grimm, H.-J., Gilfanov, M., \& Sunyaev, R. 2003, MNRAS, 339, 793

Kaur, A., Henze, M., Pietsch, W., et al. 2012, A\&A, 538, 6

Lin, D., Remillard, R. A., \& Homan, J. 2007, ApJ, 667, 1073

Lin, D., Remillard, R. A., \& Homan, J. 2009, ApJ, 696, 1257

Lin, D., Remillard, R. A., Homan, J., \& Barret, D. 2012, ApJ, 756, 34

Markowitz, A., \& Edelson, R. 2004, ApJ, 617, 939

Marshall, N., Warwick, R. S., \& Pounds, K. A. 1981, MNRAS, 194, 987

Massey, P., Olsen, K. A. G., Hodge, P. W., et al. 2006, AJ, 131, 2478

Middleton, M. J., Miller-Jones, J. C. A., Markoff, S., et al. 2013, Natur, 493, 187

Muno, M. P., Remillard, R. A., \& Chakrabarty, D. 2002, ApJL, 568, L35

Nandra, K., George, I. M., Mushotzky, R. F., Turner, T. J., \& Yaqoob, T. 1997, ApJ, 476, 70

Nooraee, N., Callanan, P. J., Barnard, R., et al. 2012, A\&A, 542, A120

Puccetti, S., Capalbi, M., Giommi, P., et al. 2011, A\&A, 528, A122

Remillard, R. A., \& McClintock, J. E. 2006, ARA\&A, 44, 49

Stanek, K. Z., \& Garnavich, P. M. 1998, ApJL, 503, L131

Stark, A. A., Gammie, C. F., Wilson, R. W., et al. 1992, ApJS, 79, 77

Stiele, H., Pietsch, W., Haberl, F., et al. 2011, A\&A, 534, A55

Stiele, H., Pietsch, W., Haberl, F., \& Freyberg, M. 2008, A\&A, 480, 599

Tananbaum, H., Peters, G., Forman, W., et al. 1978, ApJ, 223, 74

Tang, J., Yu, W.-F., \& Yan, Z. 2011, RAA, 11, 434

Vagnetti, F., Turriziani, S., \& Trevese, D. 2011, A\&A, 536, A84

van der Klis, M. 1994, ApJS, 92, 511

Voss, R., \& Gilfanov, M. 2007, MNRAS, 380, 1685

Wang, Q. D. 2004, ApJ, 612, 159

Watson, M. G., Schróder, A. C., Fyfe, D., et al. 2009, A\&A, 493, 339 